\title{
Artigos de Revisão: contribuições com enfoque em Ciência da Nutrição
}

\author{
Review articles: contributions with approach \\ in the Nutrition Science
}

Vera Lucia CHIARA ${ }^{1}$

Sonia Elza CHIARA²

\section{R E S U M O}

O objetivo deste trabalho é contribuir com a produção científica de artigos de revisão com base em pesquisas quantitativas, cuja finalidade é atualização do conhecimento sobre determinado tema, dirigindo-se especialmente aos pesquisadores iniciantes. Aborda a utilização de alguns instrumentos teórico-metodológicos para produção de textos, a priorização nos cuidados necessários quanto à leitura dos artigos consultados, o enriquecimento do conhecimento e a forma de reprodução desse conhecimento adquirido. Também alerta para os diversos aspectos que podem envolver a discussão sobre o tema escolhido, ressaltando e exemplificando a abrangência dos recursos metodológicos que precisam ser respeitados, mesmo em artigos de revisão de literatura. Conclui, orientando o compromisso com a qualidade que o desenvolvimento da pesquisa exige, e a preparação do material resultante da mesma em forma de pesquisa científica.

Termos de indexação: artigos de revisão; metodologia de pesquisa; pesquisa.

\section{A B S T R A C T}

The objective of this work is to contribute with the scientific production of review articles based on quantitative research, updating knowledge on a particular theme. This work aims specifically at beginning researchers. It covers the use of some theoretical-methodological instruments, the required caution when reading source articles, the knowledge enrichment and the proper way to expose the acquired knowledge. Additionally, it points out the many aspects involved in discussing the chosen theme, highlighting and giving examples of the scope of methodological resources that need to be respected even in literature review articles. Finally, the article advices on the commitment to quality research requires and on how to prepare and present the resulting material it in the proper scientific manner.

Indexing terms: review article; research methodological; research.

\footnotetext{
1 Departamento de Nutrição Social, Instituto de Nutrição, Universidade do Estado do Rio de Janeiro. Rua São Francisco Xavier, 524, $2^{\circ}$ andar, Sala 12001, Maracanã, 20559-900, Rio de Janeiro, RJ, Brasil. Correspondência para/Correspondence to: V.L. CHIARA.E-mails: <vchiara@easynet.com.br>; <vchiara@uerj.br>.

${ }^{2}$ Curso de Pedagogia e Curso de Formação de Professores, Escola de Educação e Meio Ambiente, Centro Universitário da Cidade do Rio de Janeiro. Rio de Janeiro, RJ, Brasil.
} 


\section{N T R O D U Ç Ã O}

O conhecimento científico tem se caracterizado, neste século, por sua velocidade de crescimento. No entanto, a rapidez na produção do saber não pode ser desacompanhada do compromisso com a qualidade dessa construção. Ao contrário, exige do pesquisador, especialmente dos principiantes, a responsabilidade que todo trabalho científico exige ${ }^{1}$.

Conhecer o funcionamento e o processo das descobertas de fenômenos estudados na Ciência da Nutrição é um desafio que se impõe na elaboração de qualquer pesquisa científica e, principalmente, no desenvolvimento de artigos de revisão de literatura para atualização do conhecimento.

Esta modalidade de estudo se constitui como pesquisa científica e implica no exercício de pensamento, reflexão, raciocínio crítico e capacidade de síntese. Para seu desenvolvimento é indispensável obedecer aos critérios científicos, para que os interessados no tema possam beneficiar-se dos resultados. Uma vez descomprometido com a aquisição do conhecimento científico, o autor pode ignorar partes importantes sobre o tema, apresentando obstáculos à compreensão da questão estudada.

Muitas vezes, o artigo se apresenta como fragmentos, partes de outros trabalhos que não se complementam e nem mesmo apresentam seqüência lógica e discussão².

A rede complexa de conhecimento racional, objetivo, metódico, sistemático, verificável, útil e comunicável, necessária a todo estudo, incluindo o de revisão de literatura, chamamos de conhecimento científico. O conhecer, a partir da revisão de artigos, possui características de atualização e reconstrução do conhecimento. A capacidade do pesquisador deve se mostrar de forma clara, precisa, objetiva e ter uma síntese inovadora do conhecimento descoberto a partir de estudos já existentes ${ }^{1}$.

Este trabalho aborda aspectos sobre o manejo de alguns instrumentos teórico-metodoló- gicos na produção de artigos de revisão, voltando-se para aqueles que têm por base pesquisas quantitativas, nas quais os tipos de delineamentos dos estudos podem ser subdivididos em descritivos e analíticos, apresentando ambos preocupações quanto à clareza do objetivo, hipóteses ou pressupostos do estudo, além de metodologia que envolva esclarecimento, viéses, precisão e acurácia ${ }^{3}$.

\section{A pesquisa bibliográfica}

A revisão de literatura está presente em toda pesquisa, sendo sempre sujeita aos aspectos que dizem respeito à questão estudada e ao tipo de estudo de revisão a ser desenvolvido. Existem diversas modalidades de trabalhos científicos em pesquisa quantitativa que empregam a revisão de literatura como base. Estas pesquisas podem ser do tipo: metanálise; revisão sistemática de literatura; baseados em evidências ou de revisão bibliográfica com o caráter eminentemente de atualização do conhecimento sobre um tema circunscrito $^{4-8}$.

No campo da Nutrição ainda são escassas as publicações destes gêneros, sendo o último tipo o mais comum e o tema central do presente estudo. A abordagem sobre estes diferentes estudos extrapola a questão deste trabalho. No entanto, em síntese, considera-se que a característica principal da metanálise refere-se à revisão aprofundada e exaustiva de artigos sob técnica que segue critérios estabelecidos a priori, dentro de rigores metodológicos próprios e exigindo desenho claramente definido para evitar viéses e erros aleatórios ${ }^{4}$. Sintetiza um processo de combinação dos resultados de diversos estudos, dos quais é possível extrair informações detalhadas, verificando se os achados da literatura são consistentes e se podem ser generalizados ${ }^{6,9}$. Outro aspecto relevante para o seu desenvolvimento diz respeito à necessidade de um conhecimento mais amplo de diversas disciplinas como epidemiologia, estatística, entre outras. Com o crescente interesse em epidemiologia nutricional, 
que estuda a relação entre a condição de saúde/ doença e o estado de nutrição, o desenvolvimento da metanálise pode, em muito, contribuir com a investigação científica, especialmente em nutrição clínica e saúde pública9 .

A terminologia "revisão sistemática de literatura" foi empregada em 1993, visando à diferenciação em relação aos estudos de metanálise. Entretanto, tal distinção ainda não é aceita por muitos estudiosos, uma vez que ambos os estudos apresentam muitas semelhanças ${ }^{5}$. Neste sentido, sugere-se que as características básicas deste tipo de pesquisa bibliográfica partem de suas premissas que visam: reduzir informações oriundas de grande quantidade de trabalhos científicos; integrar de forma crítica estes resultados; obedecer a técnicas eficientes de busca bibliográfica; avaliar a possibilidade de generalização dos dados para população, grupo de indivíduos e tratamentos clínicos específicos; analisar a consistência das relações estudadas nas pesquisas; abordar as controvérsias encontradas e elevar a precisão e acurácia dos achados ${ }^{6}$.

Outra modalidade de pesquisa científica baseada em revisão de literatura, que vem sendo amplamente difundida desde a década de 80 , define-se como pesquisa baseada em evidências ${ }^{7}$. Apresenta diversas semelhanças com a metanálise, porém, não pretende o aprofundamento extensivo e complexo que aquela impõe. Sua característica básica refere-se à contribuição da tomada de decisões na solução de determinados problemas, que dizem respeito aos métodos terapêuticos empregados, baseando-se em pesquisas metodologicamente corretas. Esta modalidade de estudo, considerada como "medicina baseada em evidências", exige o cumprimento de cuidadosa metodologia, utilizando epidemiologia clínica e bioestatística, de forma a avaliar detalhadamente os estudos selecionados, tendo em vista a aplicação dos resultados na prática profissional ${ }^{7}$.

Em Nutrição, este tipo de estudo parece especialmente adequado aos cursos que se desenvolvem na forma de residência. Sem negar as ricas fontes de conhecimentos por meio de livros, textos e artigos diversos, a proposta desse estudo encoraja a iniciativa do especialista na busca por novos conhecimentos e conseqüentes mudanças na conduta assistencial, permitindo constante análise e reavaliação da situação enfrentada e proporcionando reflexão na qualidade da assistência prestada.

A modalidade de artigos de revisão de literatura com, caráter eminentemente de atualização do conhecimento sobre um tema e objeto do presente estudo, também se sustenta em busca bibliográfica e pode ser desenvolvida tanto por meio de trabalhos em pesquisa quantitativa quanto qualitativa.

\section{Artigos de revisão para atualização do conhecimento}

Os artigos aqui considerados são habituais na área de Nutrição em conclusão de cursos de graduação, aperfeiçoamento e especialização lato-sensu ${ }^{10}$. Algumas vezes originam-se de reformulações da revisão bibliográfica, empregada no desenvolvimento de dissertações e teses em pós-graduação stricto-sensu11,12. Seu objetivo central é atualizar ou aprofundar questões sobre um tema circunscrito, normalmente discutido na atualidade, considerando o conhecimento já existente, porém aprofundando-se na busca por abordagens diferenciadas e questões controversas ${ }^{11-13}$.

No artigo de revisão desenvolvido por Baumgartner et al. ${ }^{14}$, o conhecimento existente sobre as variáveis de confundimento na associação entre indicadores antropométricos e risco de doenças crônicas (idade, sexo, etnia e outras), e ainda as limitações de alguns destes quanto às mesmas, contribuiu para a seleção dos estudos, análise e discussões destes, frente ao objetivo de associar obesidade e distribuição de gordura corporal com doenças crônicas.

Resumir um conjunto de informações originárias de diversos estudos exige meticulosa atenção por parte do pesquisador. Suckett ${ }^{15}$, ainda 
na década de 70, escrevendo sobre viéses em estudos quantitativos analíticos, como de caso-controle, apresentou extensa relação de tipos de viéses, subdivididos conforme a etapa da pesquisa. Para o autor, estes viéses podem ocorrer desde a revisão de literatura, sendo alguns relacionados ao pesquisador, de acordo com: tendência em procurar e utilizar trabalhos que apresentaram resultados positivos, em consonância com a pressuposição do próprio tema estudado; à opção em escolher artigos publicados por reconhecidos nomes de expert sobre o tema; e ao direcionamento da busca bibliográfica para uma questão específica que esteja "em moda". Esses erros provocam desvio e tendência, limitando a abordagem do tema.

Para controlar possíveis confusões e viéses, também deve ser observado o emprego de técnicas estatísticas. Concato et al. ${ }^{16}$ destacaram, de duas revistas científicas de notório reconhecimento internacional, 415 artigos, empregando modelos multivariados. O objetivo do estudo foi, justamente, demonstrar os problemas quanto ao uso desta técnica. Os autores selecionaram, dentre estes, amostras de 60 trabalhos nos quais analisaram o relato e destacaram as considerações e inadequações sobre as técnicas estatísticas empregadas.

Outro tipo de limitação metodológica foi observada em artigo de revisão, visando verificar fatores determinantes da retenção de peso em mulheres no período pós-parto ${ }^{12}$. O autor destacou que os estudos não incluíram grupos de comparação, o que contribuiria para uma análise mais apurada dos resultados obtidos, além de não relatarem suficiente caracterização das amostras quanto a faixa etária. Tendo em vista o tema do próprio estudo, o autor alertou para a necessidade de informações sobre a inclusão ou não de gestantes adolescentes nas amostras, uma vez que se reconhece a possível interferência do processo de crescimento e desenvolvimento sobre o ganho ponderal durante a gestação na fase da adolescência.

Para desenvolvimento de artigo de revisão, é importante que o pesquisador observe os termos de indexação que são empregados nos artigos consultados aspecto que, muitas vezes, facilita o aprofundamento do tema, destacando questões que anteriormente não haviam sido percebidas. Por outro lado, o relato minucioso destes termos de indexação no próprio trabalho contribui para demonstrar os detalhes metodológicos na busca bibliográfica e auxilia para que outros interessados no assunto possam buscar uma nova abordagem ${ }^{17}$. Outras questões devem ser observadas em respeito à descrição da base de dados e ao período de tempo de publicação dos artigos. Em artigo de revisão de Batista et al. ${ }^{18}$, com objetivo de estudar a relação entre a prática de atividade física durante a gestação em mulheres não atletas e o crescimento fetal, observou-se o relato das bases de dados consultadas, o tempo de publicação considerado para seleção dos artigos e os aspectos relativos à amostra, tendo em vista o interesse em gestantes não atletas.

Todas estas questões chamam atenção para a qualidade da busca bibliográfica e o cuidado na leitura.

Um dos momentos importantes no desenvolvimento de artigos de revisão se refere à relação do pesquisador com a leitura dos textos. é preciso construir e desenvolver habilidade de leitura para garantir o aproveitamento satisfatório de tais materiais. Ler proveitosamente é funda-mental para entender e ampliar o conhecimento. A leitura cuidadosa implica na capacidade de extrair informações consistentes, detalhadas e confrontáveis.

\section{A leitura científica}

É impossível pensar a utilização de um artigo como proveitoso, se a leitura não for atenta ${ }^{19}$, uma vez que esta se insere em todas as etapas do trabalho: qualidade da busca ou seja: bibliográfica; observação quanto ao rigor metodológico; aprofundamento do conhecimento; e procura por detalhes, conceitos, controvérsias e respostas. 
A primeira característica da leitura proveitosa diz respeito à clareza quanto ao objetivo do próprio estudo. Manter-se permanentemente atento sobre esta questão contribui para a seleção dos artigos e facilita as demais etapas. Este tipo de clareza foi observado no artigo de revisão de Pereira \& Koifman ${ }^{20}$. Os autores elaboraram um estudo visando contribuir para definir e sustentar o tipo de método de avaliação do consumo alimentar, que associasse o consumo pregresso com a condição de saúde do presente. Assim, a finalidade da busca bibliográfica consistiu em identificar artigos empregando métodos que avaliaram consumo de mais longo período de tempo e não o consumo do momento atual.

A segunda característica diz respeito ao vocabulário empregado nos textos. O avanço tecnológico e as descobertas permanentes no campo da Nutrição, alteram antigas denominações e trazem ainda novos termos. Não se deve ler uma frase ou parágrafo apenas para entender o sentido geral. É indispensável que se compreenda todos os aspectos abordados. Qualquer dúvida sobre o significado de palavras ou siglas precisa ser esclarecida. Estudo de revisão de literatura, desenvolvido sobre mortalidade perinatal no Brasil $^{21}$, teve o cuidado de alertar quanto à diversidade de aspectos conceituais quanto à definição e classificações empregadas sobre este tema.

A terceira característica da leitura proveitosa refere-se às regras de destaque de frases ou parágrafos nos textos consultados. É comum os principiantes marcarem grandes partes dos textos, pois consideram que tudo, ou quase tudo, é indispensável. Esta atitude pode trazer repercussões negativas na compreensão da leitura e utilização do trabalho. Primeiro, porque se o texto for realmente relevante para o estudo, raramente se recorre ao mesmo uma só vez, e se foi inadequadamente marcado torna-se difícil distinguir as partes fundamentais ao próprio trabalho. Segundo, porque com este tipo de marcação ampla haverá dificuldade para desenvolver questionamentos e síntese. Seguir algumas regras para destacar partes do texto, ajuda a localizar as idéias mestras que o estudo apresenta. E a dificuldade do principiante está, justamente, em determinar que palavras, idéias ou expressões são estas.

Para criar condições que permitam identificar o que é importante, alguns aspectos podem ser observados. É desaconselhável marcar qualquer parte do texto na primeira leitura, pois prejudica, posteriormente, distinguir as questões e parágrafos relacionados ao objeto de interesse do estudo atual em cada etapa de seu desenvolvimento. Deve ser estabelecido um tipo de marcação próprio para os diferentes aspectos de interesse e de dúvida para o estudo realizado, e pode ser colocada à margem do texto, referindo as questões sobre o tema que são abordadas naquele parágrafo. Todos estes momentos devem ser regidos pelo objetivo do estudo.

Em artigo de revisão cujo objetivo foi contribuir junto aos profissionais de nutrição com o conhecimento sobre "Amenorréia e osteoporose em adolescentes atletas" 22 , um dos principais aspectos destacado, na leitura consultada, diz respeito ao levantamento das possíveis causas dessa doença no grupo populacional de interesse. Este enfoque foi o ponto inicial apresentado no artigo de revisão dos autores. Assim, a clareza do objetivo e o cuidado na leitura irão retratar-se no próprio trabalho do pesquisador.

\section{A concretização do conhecimento}

Todo texto, seja esse um livro, capítulo ou artigo, apresenta trechos nos quais se localizam os conceitos científicos e/ou pressupostos que regem o trabalho do autor ${ }^{23}$. A identificação dos mesmos auxilia na análise e compreensão do trabalho, pois expressam a idéia principal sobre o tema desenvolvido. Estes aspectos facilitam posteriormente ao pesquisador estabelecer sua própria posição, observando os diferentes pontos de vista.

Em pesquisa quantitativa na área de nutrição, o exemplo desta questão foi observado em publicações internacionais, durante a década 
de 90, abordando os efeitos dos ácidos graxos trans sobre a saúde ${ }^{11}$. Concordâncias e discordâncias discretas, entre pesquisadores, sobre o tema foram travadas, tanto via respostas diretas quanto por meio da etapa de discussões dos resultados encontrados em suas pesquisas. Na década atual, com o crescente aumento da prevalência de obesidade, em quase todo o mundo, vem se verificando diferentes pontos de vista quanto à necessidade de controle sobre o consumo de lipídeos enquanto fator de risco associado a tal condição ${ }^{24-27}$.

O processo de observação destes detalhes enriquece o conhecimento e se revela na interpretação dos textos no artigo de revisão do pesquisador. Impede que parágrafos sejam repetidos de forma literalmente idêntica à dos artigos consultados. Este é um exercício de pensamento, reflexão e raciocínio que encoraja a iniciativa do pesquisador em interpretar com suas próprias palavras $^{28}$.

Certamente esta abordagem não se estende ao uso de citações, especialmente comuns em pesquisas qualitativas, como no artigo de Andrade \& Bosi29 nesta modalidade de pesquisa, que apresenta longas citações e obedece ao critério determinado de como expressá-las.

O interpretar concretiza o conhecimento adquirido em quantidade e qualidade ${ }^{30}$. Para emitir juízo crítico sobre a leitura, é importante que o lido seja apreendido, que os conceitos expostos se coloquem no contexto do próprio estudo, relacionando semelhanças e diferenças entre os autores consultados com as do tema abordado no artigo. O pesquisador passa a expressar a capacidade de pensar e agir segundo seus pensamentos ${ }^{31}$. Neste contexto, o artigo de revisão para atualização do conhecimento atinge seu clímax.

Sem abandonar o conhecimento existente, novos aspectos são discutidos, redesenhando as conclusões. Não mais se reporta ao que é sabido e notório no campo da ciência da Nutrição. O artigo extrapola este limite.

\section{Considerações na elaboração do artigo}

A forma de apresentação do artigo de revisão não possui um modelo rígido. De modo geral, são iniciados pela introdução, na qual se procura situar o problema, sua importância e relevância científica. Como em qualquer modalidade de artigo científico, esta etapa informa aos leitores o porquê e para quê da realização do estudo de revisão sobre o tema.

Todavia, alguns autores optam por suprimir a introdução, iniciando a apresentação de forma mais direta sobre o tema do estudo, sem que ocorra qualquer prejuízo na seqüência lógica de abordagem ao tema e compreensão do texto ${ }^{32}$. Isto porque, qualquer que seja a forma de iniciação do artigo, a questão principal que levou ao desenvolvimento do estudo deve estar clara desde o começo, complementando-se passo a passo.

O corpo do texto deve ser organizado em função da finalidade da revisão, adaptando-se à melhor maneira de reproduzir os aspectos diversos relacionados ao tema. O fundamental é organizar os parágrafos abordando pontos básicos, que permanentemente mantenham as questões conectadas de maneira mais apropriada para explanação e entendimento final.

Em dependência do objetivo e tema do estudo, quadros e tabelas podem ser incluídos, apresentando esquemas ou compilação dos trabalhos pesquisados ${ }^{20}$. As tabelas ou quadros, em algumas circunstâncias, são fundamentais para melhor demonstrar aspectos metodológicos e contradições que impediram ou contribuíram com as conclusões mais objetivas para o estudo atual. É possível desenvolver diversas modalidades de tabelas $^{33}$ e até mesmo gráficos ${ }^{34}$. Tabelas e quadros não podem ser reproduzidos sem que conste a fonte original, quando sofrem modificações frente ao modelo original, este processo deve ser relatado.

Independentemente da forma com que se desenvolve o corpo do texto, as conclusões devem, obrigatoriamente, relacionar as questões abordadas no artigo com a finalidade de seu desenvolvimento. 


\section{O N C L U S Ã O}

Para escrever um artigo de revisão exige-se o compromisso do pesquisador com a aquisição de novos conhecimentos. Conhecer é estabelecer uma relação entre o sujeito e o objeto. Representa o pesquisador que conhece o objeto de estudo, mas se propõe a adquirir um conhecimento mais aprofundado sobre o mesmo. Neste processo, o primeiro se apropria do segundo, isto é, transforma-se o objeto de estudo em um conceito, idéia ou posicionamento sobre o mesmo. Esta apropriação é comprometida com o saber científico.

A elaboração de artigos de revisão de literatura tem como compromisso a qualidade da pesquisa e o interesse por novas descobertas. Sua conclusão expressa a posição do pesquisador e se sustenta no saber científico, estando presa na relação entre o objeto de estudo, o que foi lido, compreendido, concordante e discordante, analisado e, finalmente, interpretado.

\section{REFER Ê N CIAS}

1. Rey L. Planejar e redigir trabalhos científicos. São Paulo: Edigar Blücher; 1993.

2. Moura MLS, Ferreira MC, Paine PA. Manual de elaboração de projetos de pesquisa. Rio de Janeiro: EdUERJ; 1998.

3. Zanetta DMT. Delineamento de estudos em medicina. In: Massad E, Menezes RX, Silveira PSP, Ortega NRS. Métodos quantitativos em medicina. São Paulo: Manole; 2004. p.389-422.

4. Thacker SB. Metanálisis: um enfoque cuantitativo para la integración de investigaciones. In: Publicación científica: aspectos metodológicos, éticos Y práticos en ciencias de la salud. Washington: OPS; 1994. p.187-97. Publicación Científica; 550.

5. Chalmers I, Altman DG. Systematic reviews. London: Britsh Library Group; 1995.

6. Mulrow C. Rationale for systematic review. In: Chalmers I, Altman DG. Systematic reviews. London: Britsh Library Group; 1996. p.1-8.

7. Drumond JP, Silva E. Medicina baseada em evidências. Novo paradigma assistencial e pedagógico. São Paulo: Atheneu; 1998.

8. L'Abbe KA, Detsky AS, O'Rourke K. El metanálisis em la investigación clínica. In: Publicación científica:
Aspectos metodológicos, éticos y práticos en ciencias de la salud. Washington: OPS; 1994. p.205-24. Publicación Científica; 550.

9. Freudenheim JL. Study design and hypothesis testing: issues in the evaluation of evidence from research in nutritional epidemiology ${ }^{1,2}$. Am J Clin Nutr. 1999; 69 Supplement (6):1315s-21s.

10. Chiara VL, Hasselman MH. Normas para elaboração de monografias. №1. Manual para os Cursos de Pós-Graduação lato-sensu do Instituto de Nutrição da Universidade do Estado do Rio de Janeiro. 1999. Edição revisada e ampliada. 2000. (Apostila digitada).

11. Chiara VL, Silva R, Jorge R, Brasil AP. Ácidos graxos trans: doenças cardiovasculares e saúde maternoinfantil. Rev Nutr. 2002; 15(3):341-9.

12. Kac G. Fatores determinantes da retenção de peso no pós-parto: uma revisão da literatura. Cad Saúde Pública. 2001; 17(3):455-66.

13. Marchioni DML, Slater B, Fisberg RM. Aplicações das Dietary Reference Intakes na avaliação da ingestão de nutrientes para indivíduos. Rev Nutr. 2004; 17(2):125-49.

14. Baumgartner RN, Heymsfield SB, Roche AF. Human body composition and the epidemiology of chronic disease. Obes Res. 1995; 3(1):73-95.

15. Sackett DL. Bias in analytic research. J Chr Dis. 1979; 32(1-2):51-63.

16. Concato J, Feinstein AR, Holford TR. The risk of determining risk with multivariable models. An Int Med. 1993; 118(3):201-10.

17. Moreira AF, Soares M, Follari RA, Garcia RL. Para quem pesquisamos. Para quem escrevemos. O impasse dos intelectuais. São Paulo: Cortez; 2001.

18. Batista DC, Chiara VL, Gugelmin AS, Martins PD. Atividade física e gestação: saúde da gestante não atleta e crescimento fetal. Rev Bras Saúde Mat Inf. 2003; 3(2): 151-8.

19. Ruiz JA. Metodologia Científica. Guia para eficiência nos estudos. São Paulo: Atlas; 1996.

20. Pereira RA, Koifman S. Uso do questionário de freqüência na avaliação do consumo alimentar pregresso. Rev Saúde Publica. 1999; 33(6): 610-21.

21. Fonseca SC, Coutinho ESF. Pesquisa sobre mortalidade perinatal no Brasil: revisão da metodologia e dos resultados. Cad Saúde Publica. 2004; 20(Supl 1):S7-S19.

22. Mantoanelli G, Vitalle MSS, Amancio OMS. Amenorréia e osteoporose em adolescentes atletas. Rev Nutr. 2002; 15(3):319-32.

23. Galliano AG. O método científico: teoria e prática. São Paulo: Harbra; 1986. 
24. Bray GA, Popkin BM. Dietary fat affects obesity rate. Am J Clin Nutr. 1999; 70(4):572-3.

25. Willett WC. Reply to GA Bray and BM Popkin. 1999. Am J Clin Nutr. 1999; 70(4):574.

26. Sichieri R. Is fat intake important in the public health control of obesity? Am J Clin Nutr. 2000; 72(1): 1 .

27. Bray GA, Popkin BM. Reply to R Sichieri. Am J Clin Nutr. 2000; 72(1):204-5.

28. Cervo Al, Bervan PA. Metodologia científica. São Paulo: Ms-Graw-Hill; 1983.

29. Andrade A, Bosi MLM. Mídia e subjetividade: impacto no comportamento alimentar feminino. Rev Nutr. 2003; 16(1):117-25.

30. Severino AJ. Metodologia do trabalho científico. São Paulo: Cortez e Autores Associados; 1992. p. 180.

31. Santos JA, Parra Filho D. Metodologia científica. São Paulo: Futura; 1998. p.201.
32. Pedrosa LFC, Cozzolino SMF. Alterações metabólicas e funcionais do cobre em Diabetes Mellitus. Rev Nutr. 1999; 12(3):213-24.

33. Thompson JK. Assessing body image disturbance: measure, methodology, and implementation. In: Body image, eating disorders, and obesity. An integrate guide for assessement and treatment. Washington (DC): American Psycological Association; 1996. p.10-54.

34. Heinberg AN. Theories of body image disturbance. Perceptual, development, and sociocultural factors. In: Body image, eating disorders, and obesity. An integrate guide for assessement and treatment. Washington (DC): American Psycological Association; 1996. p.101-40.

Recebido em: 27/8/2004

Versão final reapresentada em: 5/1/2005 Aprovado em: 17/3/2005 